\title{
From aliens to invisible limbs: The transitions that never make it into conscious experience
}

\author{
Jaan $\operatorname{Aru}^{1,2,3^{*}}$ \\ ${ }^{1}$ Institute of Computer Science, University of Tartu, Estonia \\ 2 Institute of Penal Law, University of Tartu, Estonia \\ ${ }^{3}$ Institute of Biology, Humboldt University Berlin, Germany \\ * corresponding author: \\ Jaan Aru \\ Email: jaan.aru@gmail.com
}

\begin{abstract}
Here it is suggested that one interesting but not well-studied property of consciousness is its continuity - the fact that my experience is stable in time despite the myriads of changes in the underlying neural activity. It is proposed that there are specific mechanisms that maintain the continuity of consciousness by preventing certain transitions in the environment from entering conscious experience. These mechanisms are the key reason why we do not perceive the involuntary eye-blinks or why our own moving limbs do not capture our attention. I will describe some studies we have conducted with virtual reality to demonstrate that one mechanism supporting the continuity of consciousness seems to be the withdrawal of attention from the specific predictable sensory activity. It is also discussed how the active inference theory can explain this set of findings. It seems that (for now) the active inference theory is the only theory that can account for the continuity of consciousness. Finally, we explore the neural mechanisms of how the motor cortex conveys specific predictions to the sensory cortices that inhibit the predicted sensory consequences of own movement. Although not much is learned from this piece about the mechanisms of continuity, it is concluded that the topic is worth to be explored more thoroughly.
\end{abstract}

\section{Understanding consciousness: where do we stand?}

Consider a lab retreat of alien scientists. They come to earth to enjoy the winter, the fact that there is only one sun, and of course they come for the ice cream. However, as they are scientists, they also find themselves thinking about humans. Surely, humans created ice cream, but alien scientists understand that this does not explain anything about the first person perspective of humans. They wonder what is it like to be a human with its consciousness. And, as they are scientists, to figure that out they turn to scientific papers.

If the alien scientists did their first exploration of human consciousness in this fashion then what would they think about human conscious experience? Would they know what it feels like for humans to have conscious experience? Would they actually have understood anything about what is it like to be conscious? 
I do not think so. For example, from the papers about global neuronal workspace theory of consciousness, they would have read that when a single stimulus is presented at the perceptual threshold, so that it sometimes is consciously perceived and sometimes not, conscious perception co-varies with the activity of the so-called frontoparietal network (to those alien scientists/visitors reading the current chapter I would suggest the following papers: Dehaene \& Naccache, 2001; Dehaene et al., 2006; Dehaene \& Changeux, 2011; Dehaene, Lau \& Kouider, 2017). They would have read that when this single stimulus is consciously perceived, neural activity starts differing from the non-conscious brain activity around $270 \mathrm{~ms}$ (Sergent, Baillet \& Dehaene, 2005; Del Cul, Baillet \& Dehaene, 2007). Hopefully, the aliens would also read that there are scientists on planet Earth who believe and have empirical evidence for saying that local activity in sensory cortices (Fisch et al., 2009) and earlier negative deflections of event-related potentials are more closely tied to consciousness (for review see Koivisto \& Revonsuo, 2010; Railo, Koivisto \& Revonsuo, 2011). Are these the key facts and debates for understanding what it is like to have human consciousness? Whether the processes that correlate with consciousness appear at 200 or $270 \mathrm{~ms}$ and whether they appear in sensory cortex or in the frontoparietal network is not crucial to understanding what is it like to have conscious experience.

More generally, the aliens would probably be rather amused about the amount of debates scientists on Earth have about consciousness. In addition to debating whether the correlates of consciousness are to be found in the frontoparietal network or locally in sensory cortex and whether they are later or earlier in time, we debate almost everything. Are the neural correlates of consciousness in the back or front of the cortex? (Boly et al., 2017; Odegaard, Knight \& Lau, 2017). Actually, we are even discussing whether the key processes constituting consciousness are in the cortex at all or is consciousness more tightly related to the activity of subcortical structures? (Panksepp, 2004; Merkel, 2007; Damasio, 2012). Is consciousness based on recurrent processing (Lamme, 2006), information integration (Tononi, Boly, Massimini \& Koch, 2016), neural synchrony (Melloni et al., 2007)? Also, we hotly debate conceptual issues. Is conscious experience rich or is it poor (Block, 2014; Lamme, 2018; Cohen, Dennet \& Kanwisher, 2016)? Is conscious perception gradual or is it all or none (Sergent et al., 2004; Overgaard et al., 2006)? Is attention independent of consciousness or is it necessary for conscious perception (Koch \& Tsuchiya, 2007; Cohen, Cavanagh, Chun \& Nakayama, 2012; Aru \& Bachmann, 2013)? There are many more debates. Of course debates are a necessary part of science, but we are discussing even the most basic things. What do we agree upon? Sometimes it seems to me that the only thing the scientists on Earth have a near-consensus on is that the mechanisms of consciousness have to be in the brain.

So why do we have so many contradictory findings and theories? We have proposed earlier that this is due to the fact that the most widely used experimental approach - contrasting trials with and without conscious perception to unravel the neural processes that differ between the conditions - is not pure. This so called contrastive analysis will always bring along neural processes that reflect unconscious prerequisites and consequences, in a manner and extent that is specific to the experimental paradigm used (Miller, 2007; Aru, Bachmann, Singer \& Melloni, 2012; De Graaf, Hsieh \& Sack, 2012). This method principally cannot reveal the true mechanisms underlying consciousness. Hence, depending on the experimental paradigm and the exact task parameters different confounding "correlates" of conscious processing appear in the results. What can we do about it? 
At least I would feel a bit embarrassed in front of my alien colleagues, as despite the fact that this problem with the contrastive method has been widely known since 2012 (Aru et al, 2012; De Graaf et al., 2012; see Miller, 2007 for an earlier treatment) and that there have been calls to arms (Aru \& Bachmann, 2015; Miller, 2015), there have been really almost no radically new experimental approaches. Indeed, the aliens could point out that in top science journals papers about consciousness are still published with the same thoroughly criticized method (Van Vugt et al. 2018). So we have endless debates and one key method that cannot resolve any of these debates. (Despite my generally pessimistic view on the progress of the science of consciousness, I would like to point out and complement several interesting approaches to tackling the problem: Frässle et al, 2014; Pitts et al., 2014; Baroni et al., 2017)

\section{Understanding consciousness: the alternative way}

After having studied consciousness with the standard contrastive method (e.g. Aru \& Bachmann, 2009a, 2009b) and with several adaptations of and improvements to it (e.g. Aru et al, 2012b; Aru et al., 2016; Rutiku, Martin, Bachmann \& Aru 2015; Rutiku et al., 2016) for many years, I personally feel that I have missed the point. I think that by being focused on "when" the activity between trials with and without consciousness diverges and "where" this activity comes from, I have missed the essence of conscious experience. So far I have always put first the neural activity that can be measured and the experimental paradigm; I think that it is time to put conscious experience first - how it feels like. In some sense this is exactly what Giulio Tononi has done: starting from the key features of consciousness (consciousness is informative and integrated), he has built a theory about what consciousness is (Tononi, 2004; Tononi et al., 2016). While I am sympathetic to the approach, I do not dare leap from the features of consciousness to a full theory. Also, I think it is very hard, if not impossible to do so (see Bayne, 2018 for a thorough critique on the axiomatic approach of Tononi). However, I do think that it is an important change to focus on the characteristics of consciousness and base the research on this. The phenomenology of conscious experience has of course been well explored by philosophers and pioneers of psychology such as Wilhelm Wundt (1874) and Edward Titchener (1907), but now we might be in a position to understand how these phenomenological qualities arise from the neural machinery. (Well, maybe not, but it seems worth trying to see how far we can take this enterprise.)

I agree with Tononi's key characteristics of consciousness (consciousness is informative and integrated, it is structured and definite; Tononi \& Koch, 2015). However, for me there is another feature that is quite curious - the continuity of consciousness in space and time. For me, this is an interesting and maybe a defining feature of conscious experience. If I lay in my armchair, thinking about aliens and consciousness, I have an experience of my surroundings that is stable in time, despite my eyes blinking and moving three times a second, causing constantly changing patterns on my retinae. For me it is mind boggling that our conscious experience is smooth and continuous despite all the transitions in the underlying neuronal activity patterns. ${ }^{1}$

Continuity of consciousness is curious. So why have we not studied this continuity? I see two reasons. First, historically the scientific study of consciousness got started through successes in comparing trials with and without conscious perception (Logothetis \& Schall, 1989). These

\footnotetext{
1 I thank Talis Bachmann for drawing my attention to this fact a decade ago; I thank Lucia Melloni for continuous discussion about continuity of consciousness.
} 
were exciting times, for instance there was a lot of buzz about $40 \mathrm{~Hz}$ oscillations (Gray et al., 1989), first fMRI studies of conscious perception were conducted (Lumer et al., 1998). With all these developments in mind sir Francis Crick wrote about the problem of consciousness "It is not impossible that, with a little luck, we may glimpse the outline of the solution before the end of the century". He meant the end of the last century, as the quote is taken from a commentary paper in 1996 (Crick, 1996). However, all of this has turned out to be more complicated (as outlined above).

The second reason is that it is much simpler to contrast trials with and without conscious perception whereas it is experimentally hard to study the mechanisms of continuity (Hohwy, 2009). For example, in all the experimental paradigms, subjects are conscious of their surroundings in both cases, with and without conscious perception of the particular target. By looking at the differences between these two conditions we are functionally subtracting away any continuity of consciousness (see Hohwy, 2009 for a longer treatment of this issue).

I think the contrastive analysis has been and will still be useful for studying consciousness. However, for me personally using advanced versions of contrastive analysis were not satisfying any more. Especially as I think that there is an interesting question to study: what grants consciousness the effortless continuity despite the restless-and-always-changing nature of the underlying neural processes? I will not pretend that one chapter can solve all the issues, but I will try to demonstrate how this shift of perspective leads to interesting questions.

\section{Why don't we perceive blinking?}

Let us first consider blindness to eye blinks as a clear example for continuity of consciousness over time despite the transitions in the retinal stimulation. The eyes blink once every few seconds, but one is completely oblivious to such shutters of experience - consciousness is continuous despite the fact that blinks cover the pupil for 100-150 ms (Riggs, 1981). At first one could object that this is really a short time, but when darkness of such length is imposed externally, one immediately notices the dark time slice (Riggs, 1981; Golan et al, 2016). So, here's a seemingly very simple yet profound demonstration: our conscious experience is continuous despite the eye-blinks. What is happening, why are we not aware of the transitions in the neural activity created by eye-blinks? Our conscious experience is maintained, but how? A key point to think about: despite the ubiquity of eye blinks, no theory of consciousness can actually explain why our experience is stable and continuous while blinking. For example, saying that consciousness is associated with the activity of the frontoparietal network does not help further: then one needs to answer why the visual cortical activity reflecting eye-blinks is not chosen for ignition in the frontoparietal network. One blinks, this leaves a strong trace on the early visual cortical activity (Golan et al, 2016), yet it does not reach consciousness. Why?

Both in the case of eye-blinks and externally induced darkness there is a change of activity on the retina and early visual areas (Golan et al, 2016), so why is this change in one case consciously experienced and in another not? Measuring cortical responses along the visual pathway it was observed that in low visual areas blinks and saccades lead to transient responses that are quite similar to externally induced darkness and image shifts (Golan et al, 2016, 2017). In contrast, high visual areas showed a steady response without the internally generated involuntary blink- and saccade-related transients. However, the activity patterns in these areas nevertheless were affected by externally induced darkness and image shifts. In 
other words, the neural activity in the high visual cortex corresponded more closely to the continuity of consciousness (Golan et al, 2016, 2017). This is not to say that such activity in higher visual cortex is the key mechanism to conscious experience, but these experiments show that somewhere along the cortical hierarchy the internally generated transitions are subtracted out.

This result by itself does not explain how exactly this continuity is achieved: what mechanism holds the steady response or suppresses the transients in higher visual cortex? The obvious difference between the eye blinks and externally induced darkness is that during eye blinks the brain can predict the change in visual stimulation and hence is able to continuously fill in the experience over time. In other words, the brain has access to its own motor commands and can take these into account for creating continuous vision (Wurtz, 2008; Golan et al, 2016). During self-generated blinks and saccades the brain can predict the changes in sensory input (Golan et al, 2016, 2017). Therefore, it is possible that this prediction is used to cancel out the transients and keep consciousness continuous.

\section{Potential mechanisms underlying continuity}

To better understand how this might work, let us consider the potential mechanisms. With regard to the cognitive level one mechanism for subtracting the transients from entering consciousness is to withdraw attention from these transitions. Attention is a powerful mechanism for boosting signals, therefore if the transition is not attended, this transition does not disrupt the continuity of consciousness. The key idea is that the brain can take its own motor commands into account for removing attention from the self-generated transients. On the other hand, externally induced transitions cannot be predicted and they hence capture attention in bottom-up fashion. This idea makes two straightforward predictions. First, if you start paying attention to your involuntary blinks you might start seeing a short flicker of your otherwise continuous conscious experience. At least from the phenomenological standpoint this seems to be true. The second prediction is that if the brain can predict the externally imposed image shifts or the timing of external darkness, then gradually over time consciousness should stay continuous despite this externally imposed darkness. In order to better understand the suggested attentional mechanism underlying the continuity of consciousness, let us first consider further examples of transitions that never make it to our consciousness.

\section{Suppressing self-generated transitions}

Eye blink and saccade related activity patterns in early sensory cortices are not the only transitions that are wiped out from conscious experience. In fact, many other movements of our body affect sensory responses in a strong fashion but do not capture attention. For example, while running, one's own hands, two relatively big objects, move through the lower part of one's visual field, without being experienced. Clearly, these two objects activate cells on the retina and the respective thalamic nuclei, but somewhere along the processing hierarchy these transients are suppressed. We know this, because while running or giving a talk our own moving hands do not capture our attention. Moreover, if there was an external object moving at the same position (a running dog, a moving ball) it would immediately capture one's attention. Our peripheral vision is very quick in spotting moving objects and directing our attention to them. However, our hands or legs moving does not bother us. 
It seems that here too the brain actively suppresses the transients and that the mechanisms for ignoring transients might be the same as in the case of eye blinks and saccades: the brain uses predictions to withdraw attention from the transients generated by itself. How could one test this conjecture experimentally?

The basic idea to test for withdrawal of attention from the consequences of own movement is relatively simple: if attention is withdrawn from the parts of the visual field where the own hand is currently moving then the reaction time (RT) to objects in those parts of the visual field should be longer. However, it is complicated to test this hypothesis with conventional tools of experimental psychology, as it would require that the participants see the moving objects "behind" the moving limb without seeing the moving limb itself. How to create invisible limbs? Virtual reality (VR) is the answer.

To quantify these intuitions that self-generated limb movements are associated with withdrawn attention we performed several experiments with VR (Laak, Vasser, Uibopuu \& Aru, 2017; Vasser, Vuillaume, Cleeremans \& Aru, 2018; See Figure 1). The participants were sitting with VR goggles on. At the same time, the participants were asked to raise their hand in front of their eyes (Figure 1A). We captured the coordinates of hand movements precisely in time and space, but in the VR environment we only presented stimuli and we did not show the hand of the participant. Hence, we could measure any effect the movement of the hand has on the stimuli that in reality would be actually behind the hand. Now we were set to understand how the self-generated hand movement modulates perception.

In our first set of experiments (Laak et al, 2017) we presented the subjects with a visual search display (inside the VR goggles): they had to spot either a small sphere moving in the opposite direction from the rest or the sphere that changed in color (Figure 1B). We measured the RT of noticing the targets. The experimental manipulation was that on half of the trials the stimuli appeared on the spot that would have been covered by the own hand (except that we did not show the hand). Would the fact, that normally this position in space would be covered by own hand movement, have any effect on the performance? Indeed, we observed that the RTs to the stimuli behind the (invisible) hand were slower than in the control conditions (Figure 1D). Hence, we concluded that the transients generated by own limb movement are subtracted from consciousness. Furthermore, as the study design (i.e. being a visual search task) resembled tasks used to study visual attention and the magnitude of the RT effect was similar to those from the classic visual search tasks, we concluded that the mechanism seems to be attentional. 



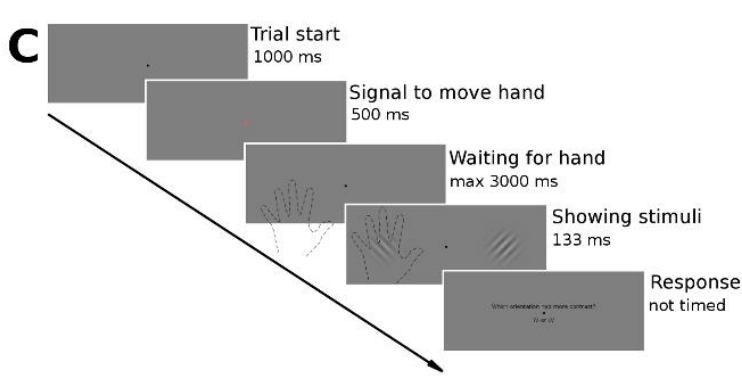

E Mean higher contrast judgements +/- SEM

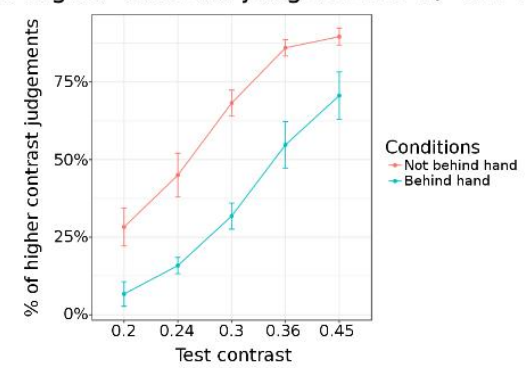

Figure 1: Self-generated movements suppress transients by withdrawing attention from them. A) Illustration of the physical setup of the experiment: The participants were sitting behind a desk and had to make a pre-trained hand movement while the hand was kept invisible in the VR environment. The coordinates of the hand were monitored and moving targets were presented behind the (invisible) moving hand. B) An illustrative view of the VR environment of Laak et al., 2017. In the critical condition, the targets were chosen from behind the (invisible) moving hand (position " $\mathrm{H} 1$ " for clarity, the hand contour, the area behind the hand, and an example random target are illustrated in green). In control conditions, the targets were shown in the area reflected from the fixation point ("Ref "). C) General design for a single trial in the experiments where visual sensitivity was tested (Vasser et al., 2019). Participants were instructed to perform a trained hand movement when the fixation cross changed color. This movement triggered the appearance of the two stimuli that were shown for $133 \mathrm{~ms}$. The task consisted in reporting the orientation of the Gabor patch with the higher contrast. Note that participants' hand was completely invisible to them. Hand outlines and Gabor patch sizes on the figure are illustrative. D) The mean RTs in Experiment 1 of Laak et al (2017) for both conditions from the panel B. The participants had to react as fast as possible to moving targets either in an area currently covered by the (invisible) hand ("Behind hand", illustrated as " $\mathrm{H} 1$ " on panel B) or a symmetric area in the other side of the visual field ("Reflected"; illustrated as "Ref on panel B). The RTs in the experimental condition ("Behind hand") were slower than in the control condition ("Reflected"). E) Results of Experiment 1 of Vasser et al (2019). X axis is the value of the test contrast, $Y$ axis denotes the percentage of trials that were reported as higher in contrast. Blue line shows the target stimulus appearing behind the hand and red line shows the target stimulus not behind the hand.

However, in this initial work we could not demonstrate that these self-generated transitions indeed are suppressed from conscious experience. This is because the RTs are a notoriously bad measure of perceptual experience. Hence, the key question remained: does this withdrawal of attention indeed also have perceptual consequences? In the follow up study (Vasser et al., 2019) we wanted to assess whether self-generated hand movement indeed changes perception. We based our study on the experimental approach used by Carrasco and colleagues who had elegantly demonstrated that attention affects perceptual experience (Carrasco, Ling \& Read, 2004). In that paradigm, two Gabor patches with different orientations 
are presented, one cued and the other not, and the subjects are asked for the orientation of the Gabor with the stronger contrast (Figure 1C). With this experimental approach it was possible for Carrasco and colleagues to demonstrate that covert attention enhances the perceived contrast of an attended stimulus (Carrasco et al., 2004). We reasoned that if 1) attention is withdrawn from the part of the visual field where the hand is moving (Laak et al., 2017) and 2) the deployment of attention affects perceived contrast (Carrasco et al., 2004), then the subjective contrast of objects in the region of the visual field where the hand is moving ought to be reduced. Hence, we performed a study with two Gabors in our VR setting (Figure 1C). After initiating the hand movement, two Gabors were presented and the participants had to indicate the orientation of the Gabor patch with higher contrast. We observed that the perceived contrast of the stimulus behind the invisible hand was significantly reduced (Figure $1 \mathrm{E})$. We replicated these results in an independent group of participants. This result again lends support for the claim that attention is withdrawn from the sensory transients generated by the brain itself. More importantly, this result shows that such self-generated transients are attenuated from experience.

To sum up the last parts: there seem to be mechanisms for actively hindering information from entering consciousness. These phenomena unravel how the brain is avoiding transitions in consciousness despite transitions in the underlying neural activity. The key mechanism for this seems to be the withdrawal of attention from these transients, where this withdrawal is enabled through the predictability of the stimulus. Can this line of reasoning bring us any closer to understanding consciousness?

\section{Intrinsic control of attention and theories of consciousness}

Continuity of consciousness seems to be one of the key characteristics of our conscious experience. Yet, most of the theories of consciousness are only concerned with what happens to stimuli that are presented in the environment (mostly a computer screen). Or, in other words: no theory of consciousness can easily explain why and how eye blinks, saccades or other self-generated movements are suppressed from consciousness. Continuity of consciousness is not in the focus of the theories of consciousness.

Interestingly, a particular set of computational theories that are not especially designed for explaining consciousness can readily explain the attenuation of sensory transients. In some sense this is paradoxical: why should a theory that is not meant for explaining conscious experience do a better job in explaining phenomena of conscious experience than theories of consciousness? One could also say that this is not paradoxical, but just reflects the sad state of consciousness research.

The active inference, or prediction error minimization theory (Friston, 2010; Hohwy, 2013; Clark, 2015) posits that the top-down and bottom-up information flow across the hierarchical structure of the cortex implements hierarchical probabilistic inference about the causes of sensory data. Within this framework predictions (or beliefs) represent the hidden causes in the world that cause sensory states in the cortex. Prediction errors signal the discrepancy between the incoming sensory data and the predictions. Prediction errors are weighted by their precision. Precision determines how strongly prediction errors influence the updating of prior beliefs. According to the most widely known implementation of predictive processing the main goal of neural computation is to minimize the (precision weighted) prediction error in the long 
term (Friston, 2010; Hohwy, 2013; Clark, 2015). Within this theoretical framework, performing actions is an efficient way of minimizing prediction errors by changing the sensory data to fit the predictions (Friston, 2010; Hohwy, 2013; Clark, 2015).

Crucially for the present chapter, the active inference theory suggests that movements are elicited by predicting the sensory consequences of the movement (e.g. the prediction that the hand will be moving in the visual field is the trigger for the movement). These predictions will drive the behavior so that the organism will perform movements that lead to the predicted state. However, the predicted consequences of movement are not in agreement with the current sensory data (where the hand is still in a resting position). Hence, there is a tension within the system between the prediction ("hand is moving") and sensory data ("hand is not moving"). According to the active inference theory, this tension is solved by reducing the precision of the current sensory data. This theoretical framework says that such reduction is the basis for sensory attenuation and that this reduction of the precision is necessary for allowing the movement to unfold (Brown et al., 2013. Therefore, this theory explains sensory attenuation as a necessary consequence of movement: without sensory attenuation movement could not happen (Brown et al., 2013). As attention is the key mechanism for regulating the precision of the prediction errors (Feldman \& Friston, 2010; Hohwy, 2013; Clark, 2015) the active inference theory proposes that the precision is reduced through the withdrawal of attention from the current sensory prediction errors. This theory might sound counterintuitive, even unnatural (for a longer treatment see Clark, 2015), but importantly for the present purposes, sensory attenuation of self-generated transients is a central part of this theoretical framework. Unlike theories of consciousness, this general computational theory can readily explain why self-generated transients are suppressed from conscious experience. In this sense, the continuity of consciousness follows naturally from this framework.

However, are we now any closer to understanding what consciousness is? There are several perspectives on how active inference could explain consciousness (Howhy, 2013; Clark, 2015; Seth, 2015; Friston, 2018). I am personally reluctant to say that active inference is the best framework for understanding consciousness, but I would conclude that active inference although not originally being a theory of consciousness - is a viable alternative to the currently prominent theories of consciousness.

Anyhow, for me the key interest has always been to understand not the computational theory behind consciousness, but the neural constituents of consciousness (Aru et al., 2012a; Miller, 2015). Does this speculation about the continuity of consciousness tell us anything meaningful about the neural processes underlying conscious experience?

\section{What is the neural mechanism for cancelling out transitions?}

What are the neural mechanisms for cancelling out sensory transients generated by own movement? In the last years these mechanisms have been worked out especially in mice, where technologies allow one to track and manipulate specific neural pathways. Hence, it has been established that motor cortex sends information even to primary visual (Leinweber et al., 2017) and auditory (Nelson et al., 2013; Schneider, Sundararajan \& Mooney, 2018) cortex. For example in the primary auditory cortex the axons from motor cortex have excitatory synapses but exert a primarily suppressive effect, because they also make synapses to inhibitory neurons (Nelson et al., 2013; Schneider et al., 2018). This pathway from motor 
cortex to sensory cortex is strong and connects to neurons in all cortical layers of the sensory cortex (Nelson et al., 2013; Leinweber et al., 2017). Hence, this projection from the motor cortex to the sensory cortex has the desired properties to reduce the effect of self-generated sensory transients: a) information about own motor commands can be sent from motor cortex to the sensory cortex and $b$ ) the net-effect of this projection is to inhibit the incoming sensory data, making the animal less sensitive to self-generated transients.

To give one particular example, David Schneider and colleagues (Schneider et al., 2018) designed an "acoustic virtual reality system" for the mice. The idea of this setup was similar to the above described experiments with VR, only the association between the self-generated movement and its sensory consequences was not learned over the lifetime of the mice, but during the course of the experiment. In this setup the mice were running on a treadmill and the scientists played the mice a series of tones whose rate was proportional to the running speed of mice. The advantage of doing these VR experiments in mice is that one can also monitor and manipulate the animals' neural activity, which allowed the authors to identify the neural circuit mechanisms that learn to suppress movement-related sounds. David Schneider and colleagues (Schneider et al., 2018) found that over the course of learning the connections from motor cortex to specific inhibitory neurons in the auditory cortex are strengthened so that the sounds associated to the treadmill running are selectively cancelled out. The authors proceeded to demonstrate that this learned inhibition of sensory signals leads to decreased sensitivity in a tone detection task, such that tones associated to movement are detected worse than tones not associated to movement. This finding nicely parallels the results obtained in human VR studies, where self-generated movement led to decreased sensitivity of visual information (Vasser et al., 2019). Therefore, we consider it to be plausible that this selective inhibition sent from the motor cortex to the sensory cortex is also the neural basis of the findings obtained in human VR studies.

In summary, studies with rodents have demonstrated that neural inhibition actively works in the background to keep predicted content from being processed further. To phrase it within the context of the present chapter I suggest that inhibition governs the continuity of conscious perception. In particular I propose that if the brain can predict self-generated transients (e.g. blinks, eye movements, limb movements), it will inhibit the specific sensory pathways that would process this sensory information. One could say that this is the neural mechanism of "withdrawn attention", but an alternative framing would be to say that the respective parts of the cortex are briefly disconnected from the world: inhibition cuts the specific parts of the cortex off from the input. Hence, from the perspective of the processing stages downstream from the populations receiving this inhibition it will look as if the transient never happened.

\section{What is the mechanism of consciousness?}

At first, it might seem that knowing the neural mechanisms of how self-generated transients are prohibited from entering consciousness will not bring us closer to understanding the neural mechanisms of consciousness. However, I personally feel that this line of thinking about consciousness does give us another angle to study the neural mechanisms of consciousness. Namely, from the current perspective we might want to understand those neural processes that co-vary with the continuity of consciousness. The main difference to the approach that is currently used is that more focus should be on trying to study those neural processes that stay the same despite the transitions (in cases where conscious experience stays continuous and 
also does not reflect these transitions). If consciousness is continuous and is not updated by a transition in the environment, the neural constituting mechanisms of consciousness should also stay oblivious or ignorant of this transition. Which neural processes are the ones that stay the same despite the transitions?

Golan and colleagues (Golan et al, 2016, 2017) had observed that in higher visual areas the self-generated transients (eye-blinks and saccades) do not influence the activity. Hence, higher visual areas never witness the transients: for them this did not happen. However, this does not necessarily mean that these higher order sensory areas are the sole or the primary loci related to conscious experience. For example, the cortex is tightly connected to the higherorder nuclei of the thalamus (e.g. Halassa \& Kastner, 2017). If the respective sensory cortical area is inhibited and never sends information on to these thalamic nuclei, continuity of whatever process is running there can be maintained. Hence, the present ideas area also compatible with theoretical views that put the thalamus in the center stage for consciousness (e.g. Bachmann, 1984; Ward, 2011). In any case I think we should seek for these neural mechanisms whose activity remains continuous when conscious experience is continuous despite the transitions of stimuli.

\section{Going forward}

Studying consciousness is hard, but sometimes I feel this "hardness" has made us - the researchers - lazy. We are still using the same experimental paradigms that were applied when the scientific study of consciousness was started (with some minor and some important modifications, of course). These experimental paradigms have fundamental problems that cannot be fixed by simply tinkering with them. I hence think that it is time to turn our attention to experimental ideas that are different. In this chapter, I favored the position that instead of looking what is the difference between trials with and without conscious perception, one could study what grants consciousness its continuity.

\section{Acknowledgements}

I would like to thank Talis Bachmann, Lucia Melloni and Renate Rutiku for having supported me with ideas and discussions over many years. I am indebted to Madis Vasser, Laurene Vuillaume and Kristjan-Julius Laak for conducting the virtual reality experiments. 


\section{References}

Aru, J., \& Bachmann, T. (2009a). Occipital EEG correlates of conscious awareness when subjective target shine-through and effective visual masking are compared: Bifocal early increase in gamma power and speed-up of P1. Brain research, 1271, 60-73.

Aru, J., \& Bachmann, T. (2009b). Boosting up gamma-band oscillations leaves target-stimulus in masking out of awareness: explaining an apparent paradox. Neuroscience letters, 450(3), 351-355.

Aru, J., \& Bachmann, T. (2013). Phenomenal awareness can emerge without attention. Frontiers in Human Neuroscience, 7,891 .

Aru, J., \& Bachmann, T. (2015). Still wanted-the mechanisms of consciousness!. Frontiers in psychology, 6, 5.

Aru, J., Bachmann, T., Singer, W., \& Melloni, L. (2012a). Distilling the neural correlates of consciousness. Neuroscience \& Biobehavioral Reviews, 36(2), 737-746.

Aru, J., Axmacher, N., Do Lam, A. T., Fell, J., Elger, C. E., Singer, W., \& Melloni, L. (2012b). Local categoryspecific gamma band responses in the visual cortex do not reflect conscious perception. Journal of Neuroscience, 32(43), 14909-14914.

Aru, J., Rutiku, R., Wibral, M., Singer, W., \& Melloni, L. (2016). Early effects of previous experience on conscious perception. Neuroscience of Consciousness, 2016(1).

Bachmann, T. (1984). The process of perceptual retouch: Nonspecific afferent activation dynamics in explaining visual masking. Perception \& Psychophysics, 35(1), 69-84.

Baroni, F., van Kempen, J., Kawasaki, H., Kovach, C. K., Oya, H., Howard, M. A., ... \& Tsuchiya, N. (2017). Intracranial markers of conscious face perception in humans. NeuroImage, 162, 322-343.

Bayne, T. (2018). On the axiomatic foundations of the integrated information theory of consciousness. Neuroscience of consciousness, 2018(1), niy007.

Blakemore, S. J., Wolpert, D. M., \& Frith, C. D. (1998). Central cancellation of self-produced tickle sensation. Nature neuroscience, 1(7), 635.

Boly, M., Massimini, M., Tsuchiya, N., Postle, B. R., Koch, C., \& Tononi, G. (2017). Are the neural correlates of consciousness in the front or in the back of the cerebral cortex? Clinical and neuroimaging evidence. Journal of Neuroscience, 37(40), 9603-9613.

Block, N. (2014). Rich conscious perception outside focal attention. Trends in Cognitive Sciences, 18(9), 445447.

Brown, H., Adams, R. A., Parees, I., Edwards, M., \& Friston, K. (2013). Active inference, sensory attenuation and illusions. Cognitive processing, 14(4), 411-427.

Carrasco, M., Ling, S., \& Read, S. (2004). Attention alters appearance. Nature neuroscience, 7(3), 308.

Clark, A. (2015). Surfing uncertainty: Prediction, action, and the embodied mind. Oxford University Press.

Cohen, M. A., Cavanagh, P., Chun, M. M., \& Nakayama, K. (2012). The attentional requirements of consciousness. Trends in cognitive sciences, 16(8), 411-417. 
Cohen, M. A., Dennett, D. C., \& Kanwisher, N. (2016). What is the bandwidth of perceptual experience?. Trends in cognitive sciences, 20(5), 324-335.

Damasio, A. (2012). Self comes to mind: Constructing the conscious brain. Vintage.

Dehaene, S., \& Changeux, J. P. (2011). Experimental and theoretical approaches to conscious processing. Neuron, 70(2), 200-227.

Dehaene, S., Changeux, J. P., Naccache, L., Sackur, J., \& Sergent, C. (2006). Conscious, preconscious, and subliminal processing: a testable taxonomy. Trends in cognitive sciences, 10(5), 204-211.

Dehaene, S., Lau, H., \& Kouider, S. (2017). What is consciousness, and could machines have it?. Science, 358(6362), 486-492

Dehaene, S., \& Naccache, L. (2001). Towards a cognitive neuroscience of consciousness: basic evidence and a workspace framework. Cognition, 79(1-2), 1-37.

Del Cul, A., Baillet, S., \& Dehaene, S. (2007). Brain dynamics underlying the nonlinear threshold for access to consciousness. PLoS biology, 5(10), e260.

De Graaf, T. A., Hsieh, P. J., \& Sack, A. T. (2012). The 'correlates' in neural correlates of consciousness. Neuroscience \& Biobehavioral Reviews, 36(1), 191-197.

Fisch, L., Privman, E., Ramot, M., Harel, M., Nir, Y., Kipervasser, S., ... \& Malach, R. (2009). Neural "ignition": enhanced activation linked to perceptual awareness in human ventral stream visual cortex. Neuron, 64(4), 562-574.

Friston, K. (2010). The free-energy principle: a unified brain theory?. Nature Reviews Neuroscience, 11(2), 127.

Friston, K. (2018). Am I Self-Conscious? (Or Does Self-Organization Entail Self-Consciousness?). Frontiers in psychology, 9.

Frässle, S., Sommer, J., Jansen, A., Naber, M., \& Einhäuser, W. (2014). Binocular rivalry: frontal activity relates to introspection and action but not to perception. Journal of Neuroscience, 34(5), 1738-1747.

Gray, C. M., König, P., Engel, A. K., \& Singer, W. (1989). Oscillatory responses in cat visual cortex exhibit inter-columnar synchronization which reflects global stimulus properties. Nature, 338(6213), 334.

Golan, T., Davidesco, I., Meshulam, M., Groppe, D. M., Mégevand, P., Yeagle, E. M., ... \& Deouell, L. Y., Mehta, A.D., Malach, R. (2016). Human intracranial recordings link suppressed transients rather than 'filling-in' to perceptual continuity across blinks. Elife, 5, e17243.

Golan, T., Davidesco, I., Meshulam, M., Groppe, D. M., Mégevand, P., Yeagle, E. M., ... \& Deouell, L. Y. (2017). Increasing suppression of saccade-related transients along the human visual hierarchy. eLife, 6, e27819

Hohwy, J. (2009). The neural correlates of consciousness: new experimental approaches needed?. Consciousness and cognition, 18(2), 428-438.

Hohwy, J. (2013). The predictive mind. Oxford University Press. 
Halassa, M. M., \& Kastner, S. (2017). Thalamic functions in distributed cognitive control. Nature neuroscience, 20(12), 1669-1679.

Koch, C., \& Tsuchiya, N. (2007). Attention and consciousness: two distinct brain processes. Trends in cognitive sciences, 11(1), 16-22

Koivisto, M., \& Revonsuo, A. (2010). Event-related brain potential correlates of visual awareness. Neuroscience \& Biobehavioral Reviews, 34(6), 922-934.

Laak, K. J., Vasser, M., Uibopuu, O. J., \& Aru, J. (2017). Attention is withdrawn from the area of the visual field where the own hand is currently moving. Neuroscience of Consciousness, 3(1).

Lamme, V. A. (2006). Towards a true neural stance on consciousness. Trends in cognitive sciences, 10(11), 494-501

Lamme, V. A. (2018). Challenges for theories of consciousness: seeing or knowing, the missing ingredient and how to deal with panpsychism. Phil. Trans. R. Soc. B, 373(1755), 20170344.

Leinweber, M., Ward, D. R., Sobczak, J. M., Attinger, A., \& Keller, G. B. (2017). A sensorimotor circuit in mouse cortex for visual flow predictions. Neuron, 95(6), 1420-1432.

Logothetis, N. K., \& Schall, J. D. (1989). Neuronal correlates of subjective visual perception. Science, 245(4919), 761-763.

Lumer, E. D., Friston, K. J., \& Rees, G. (1998). Neural correlates of perceptual rivalry in the human brain. Science, 280(5371), 1930-1934.

Melloni, L., Molina, C., Pena, M., Torres, D., Singer, W., \& Rodriguez, E. (2007). Synchronization of neural activity across cortical areas correlates with conscious perception. Journal of neuroscience, 27(11), 2858-2865.

Miller, S. M. (2007). On the correlation/constitution distinction problem (and other hard problems) in the scientific study of consciousness. Acta Neuropsychiatrica, 19(3), 159-176.

Miller, S. M. (Ed.). (2015). The Constitution of Phenomenal Consciousness: Toward a science and theory (Vol. 92). John Benjamins Publishing Company.

Nelson, A., Schneider, D. M., Takatoh, J., Sakurai, K., Wang, F., \& Mooney, R. (2013). A circuit for motor cortical modulation of auditory cortical activity. Journal of Neuroscience, 33(36), 14342-14353.

Odegaard, B., Knight, R. T., \& Lau, H. (2017). Should a few null findings falsify prefrontal theories of conscious perception?. Journal of Neuroscience, 37(40), 9593-9602.

Overgaard, M., Rote, J., Mouridsen, K., \& Ramsøy, T. Z. (2006). Is conscious perception gradual or dichotomous? A comparison of report methodologies during a visual task. Consciousness and cognition, 15(4), 700-708.

Panksepp, J. (2004). Affective neuroscience: The foundations of human and animal emotions. Oxford university press.

Pitts, M. A., Metzler, S., \& Hillyard, S. A. (2014). Isolating neural correlates of conscious perception from neural correlates of reporting one's perception. Frontiers in psychology, 5, 1078. 
Railo, H., Koivisto, M., \& Revonsuo, A. (2011). Tracking the processes behind conscious perception: a review of event-related potential correlates of visual consciousness. Consciousness and cognition, 20(3), 972-983.

Rutiku, R., Aru, J., \& Bachmann, T. (2016). General markers of conscious visual perception and their timing. Frontiers in human neuroscience, 10, 23.

Rutiku, R., Martin, M., Bachmann, T., \& Aru, J. (2015). Does the P300 reflect conscious perception or its consequences?. Neuroscience, 298, 180-189.

Schneider, D. M., Sundararajan, J., \& Mooney, R. (2018). A cortical filter that learns to suppress the acoustic consequences of movement. Nature, 561(7723), 391.

Sergent, C., \& Dehaene, S. (2004). Is consciousness a gradual phenomenon? Evidence for an all-or-none bifurcation during the attentional blink. Psychological science, 15(11), 720-728.

Sergent, C., Baillet, S., \& Dehaene, S. (2005). Timing of the brain events underlying access to consciousness during the attentional blink. Nature neuroscience, 8(10), 1391.

Titchener, E. B. (1907). An outline of psychology. Macmillan.

Tononi, G. (2004). An information integration theory of consciousness. BMC neuroscience, 5(1), 42.

Tononi, G., Boly, M., Massimini, M., \& Koch, C. (2016). Integrated information theory: from consciousness to its physical substrate. Nature Reviews Neuroscience, 17(7), 450.

Tononi, G., \& Koch, C. (2015). Consciousness: here, there and everywhere?. Phil. Trans. R. Soc. B, 370(1668), 20140167

Tsuchiya, N., Wilke, M., Frässle, S., \& Lamme, V. A. (2015). No-report paradigms: extracting the true neural correlates of consciousness. Trends in cognitive sciences, 19(12), 757-770.

Van Vugt, B., Dagnino, B., Vartak, D., Safaai, H., Panzeri, S., Dehaene, S., \& Roelfsema, P. R. (2018). The threshold for conscious report: Signal loss and response bias in visual and frontal cortex. Science, 360(6388), $537-542$

Vasser, M., Vuillaume, L., Cleeremans, A., \& Aru, J. (2019). Waving goodbye to contrast: Self-generated hand movements attenuate visual sensitivity. Neuroscience of consciousness, 2019(1), niy013.

Ward, L. M. (2011). The thalamic dynamic core theory of conscious experience. Consciousness and Cognition, 20(2), 464-486.

Wundt, W. M. (1874). Grundzüge de physiologischen Psychologie (Vol. 1). W. Engelman.

Wurtz, R. H. (2008). Neuronal mechanisms of visual stability. Vision research, 48(20), 2070-2089. 